\title{
Robotic Surgery is a Successful Tool for the Management of Large Retroperitoneal Tumors in Children
}

\section{Xavier Delforge, Anthony Kallas-Chemaly and Henri Steyaert}

Department of Pediatric Surgery, Hôpital Universitaire des Enfants Reine Fabiola, Université Libre de Bruxelles, ULB, Brussels, Belgium

Corresponding author: Xavier Delforge, Department of Pediatric Surgery, Hôpital Universitaire des Enfants Reine Fabiola, Université Libre de Bruxelles, ULB, Brussels, Belgium, Tel: 33609975574; E-mail:xdelforge@gmail.com

Received: 17 June 2016; Accepted: 01 August 2016; Published: 04 August 2016

Citation: Delforge X, Kallas-Chemaly A, Steyaert H. Robotic Surgery is a Successful Tool for the Management of Large Retroperitoneal Tumors in Children. Arch Can Res. 2016, 4: 3.

\section{Case Blog}

A 2 years old boy was admitted in our institution for abdominal mass. He presented a growing abdominal mass, associated with constipation, and recently complained of pain with walking. The computed tomography-scan showed a large mass of the retroperitoneum (Figure 1), compressing the right ureter with upper urinary tract dilation (Figure 2). The alphafoetoprotein (AFP) blood level was $7500 \mathrm{ng} / \mathrm{mL}$ [1].

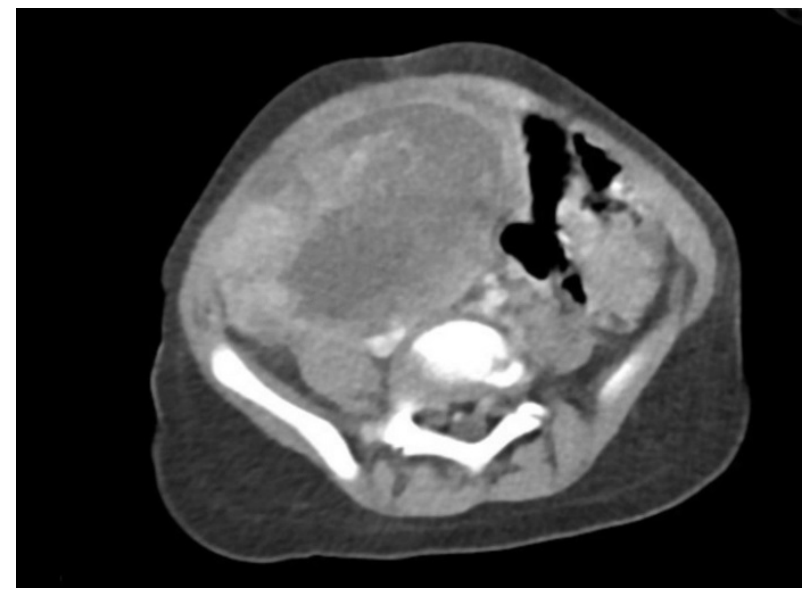

Figure 1 Computed tomography showing a large mass of the right retroperitoneum.

The patient was treated for a germ cell tumor, according to the TGM2013 protocol. He received chemotherapy first, with 4 cures associating Vinblastin, Bleomycin, and Cisplatin. The AFP level normalized after chemotherapy. The magnetic resonance imaging evaluation showed an $80 \%$ reduction of the mass. The tumor measured $20 \times 27 \mathrm{~mm}$, located in the right retroperitoneum, with close relationships with the ureter and the iliac vessels.

The surgical resection was indicated [2,3]. Laparoscopy is the approach giving the simpler postoperative outcome. Giving the size and the location of the tumor, robotic surgery was the option allowing laparoscopy. The articulated tools and the tridimensional view made possible the delicate dissection of the ureter and the iliac vessels.

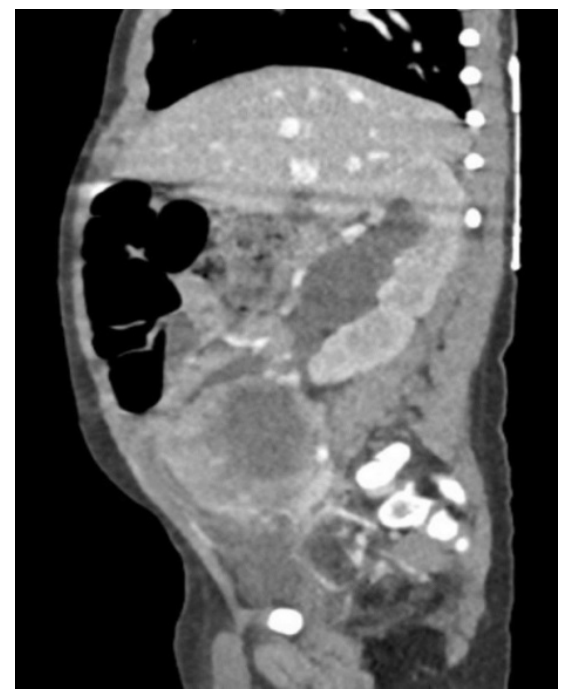

Figure 2 Computed tomography: Sagittal view revealing the ureteral compression and the upper urinary tract dilation.

The pathology confirmed the total resection of a germ cell tumor. The post-operative AFP level remained normal. The patient had a simple immediate outcome, and returned home three days after surgery.

\section{References}

1. Blohm ME, Vesterling-Hörner D, Calaminus G, Göbel U (1998) Alpha 1-fetoprotein (AFP) reference values in infants up to 2 years of age. Pediatr Hematol Oncol 15: 135-142.

2. Ahmed HU, Arya M, Muneer A, Mushtaq I, Sebire NJ (2010) Testicular and paratesticular tumours in the prepubertal population. Lancet Oncol 11: 476-483.

3. Martelli H, Patte C (2003) Tumeurs des gonades chez l'enfant. Arch Pédiatrie 10: 246-250. 